\title{
High developmental pluripotency-associated 4 expression promotes cell proliferation and glycolysis, and predicts poor prognosis in non-small-cell lung cancer
}

\author{
LONGFEI LI $^{1 *}$, YUFENG WANG ${ }^{2 *}$, QIANG WANG ${ }^{3 *}$, JINGMING QU $^{1}$, XIANGJU WEI $^{1}$, \\ JILEI XU ${ }^{1}$, YUANJIN WANG ${ }^{1}$, FENG SUO $^{1}$ and YANGJIE ZHANG ${ }^{4}$ \\ Departments of ${ }^{1}$ Cardiothoracic Surgery, ${ }^{2}$ Nuclear Medicine and ${ }^{3}$ Radiotherapy and Oncology, \\ Xuzhou Cancer Hospital, Xuzhou, Jiangsu 221000; ${ }^{4}$ Department of Orthopedics, Yunnan Cancer Hospital, \\ The Third Affiliated Hospital of Kunming Medical University, Kunming, Yunnan 650118, P.R. China
}

Received September 14, 2018; Accepted March 15, 2019

DOI: $10.3892 / \mathrm{mmr} .2019 .10272$

\begin{abstract}
The developmental pluripotency-associated 4 (Dppa4) gene serves critical roles in cell self-renewal, as well as in cancer development and progression. However, the regulatory role of Dppa4 in non-small-cell lung cancer (NSCLC) and its underlying mechanisms remain elusive. The aim of the present study was to investigate the biological function of Dppa4 in NSCLC and its underlying mechanism of action. Dppa4 expression was measured in NSCLC tissue samples and cell lines, and its effect on cell proliferation and the expression of glycolytic enzymes was determined. In addition, the underlying mechanisms of Dppa4-induced alterations in glycolysis were analyzed. Univariate and multivariate analyses were also performed to analyze the prognostic significance of clinicopathological characteristics. Dppa4 was found to be highly expressed in NSCLC tissues and cell lines. Furthermore, it was observed that Dppa4 was correlated with the degree of tumor differentiation and TNM stage. Univariate and multivariate analyses identified Dppa4 expression and clinical stage as prognostic factors for NSCLC patients. Kaplan-Meier analysis further revealed that patients with lower Dppa4 expression exhibited a better prognosis. In NSCLC cells, Dppa4 knockdown inhibited cell proliferation, while Dppa4 overexpression enhanced cell proliferation, which was likely mediated by glycolysis promotion. Dppa4 knockdown had no evident effect on the majority of enzymes examined; however, glucose
\end{abstract}

Correspondence to: Dr Yangjie Zhang, Department of Orthopedics, Yunnan Cancer Hospital, The Third Affiliated Hospital of Kunming Medical University, 519 Kun Zhou Road, Kunming, Yunnan 650118, P.R. China

E-mail: ynzlzyj@tom.com

\section{${ }^{*}$ Contributed equally}

Key words: non-small-cell lung cancer, developmental pluripotency-associated 4, prognosis, glycolysis, lactate dehydrogenase B transporter type 4 (GLUT-4) and pyruvate kinase isozyme M2 were significantly upregulated, and hexokinase II (HK-II) and lactate dehydrogenase B (LDHB) were downregulated following Dppa4 knockdown. By contrast, Dppa4 overexpression resulted in downregulation of GLUT-4, and upregulation of HK-II, enolase and LDHB, whereas it had no effect on other enzymes. Since the most evident effect was observed on LDHB, further functional experiments demonstrated that this enzyme reversed the promoting effects of Dppa4 in NSCLC. In conclusion, Dppa4 promotes NSCLC progression, partly through glycolysis by LDHB. Thus, the Dppa4-LDHB axis critically contributes to glycolysis in NSCLC cells, thereby promoting NSCLC development and progression.

\section{Introduction}

Non-small-cell lung cancer (NSCLC) accounts for $\sim 80 \%$ of all lung cancer cases according to its pathological classification (1). Although advances in diagnostic and therapeutic techniques have improved the early detection and reduced the mortality rate of lung cancer, it remains the leading cause of cancer-associated mortality worldwide (1). Thus, it is imperative to elucidate the mechanism underlying the development and progression of NSCLC.

Cancer cells and embryonic stem (ES) cells have long been known to share common characteristics with respect to self-renewal, proliferation and indefinite growth (2). Developmental pluripotency-associated 4 (Dppa4) is highly expressed in ES cells. In addition, self-renewal regulatory factors, such as Oct4, Sox2, Bmi and Nanog, are highly expressed in tumor cells and serve an important role in carcinogenesis $(3,4)$. The Dppa gene family is a cluster of five genes whose developmental expression patterns are similar to those of Oct4 (5). The structure and functions of Dppa4 indicate its possible involvement in cancer progression. Dppa4 has been found to be highly expressed in colon, prostate and bladder carcinomas, and may be a novel predictor of prognosis and a potential therapeutic target in colon cancer $(4,6)$. It has also been reported that Dppa4 functioned as an oncogene in mouse 3T3 cells and immortalized human dermal fibroblasts, 
and enhanced cell proliferation by inducing G1/S arrest (7). However, the clinicopathological significance of Dppa4, and its possible mechanism in NSCLC tumorigenesis and progression remain unclear.

The aim of the present study was to explore the expression pattern of Dppa4 in NSCLC tissue samples, including squamous cell carcinoma, adenocarcinoma, large cell carcinoma, adenosquamous carcinoma and other cell lines in order to determine whether the overexpression of Dppa4 is associated with unfavorable clinicopathological variables. In addition, the study assessed whether Dppa4 may be of value as a novel prognostic marker for NSCLC. The possible action mechanism of Dppa4 in NSCLC was also investigated. The results revealed that Dppa4 promoted NSCLC by regulating glycolysis, possibly through LDHB.

\section{Materials and methods}

Human tissue specimens. A total of 100 tumor tissue samples and 100 adjacent normal tissue samples were collected from the Department of Cardiothoracic Surgery, Xuzhou Cancer Hospital (Xuzhou, China) between January 2010 and December 2014. The tissue samples included squamous cell carcinoma, lung adenocarcinoma, large cell carcinoma and adenosquamous carcinoma. The mRNA expression of Dppa4 was evaluated in 20 pairs of fresh NSCLC tissues and adjacent normal tissues using reverse transcription-quantitative PCR (RT-qPCR), while a total of 80 paired tissue samples were embedded in paraffin to examine the Dppa4 protein levels via immunohistochemistry (IHC). All the patients had been diagnosed with NSCLC, which was confirmed by histological examination. Patients who had previously received radiotherapy or chemotherapy were excluded. The study protocol was approved by the Research Ethics Committee of Xuzhou Cancer Hospital, and informed consent was obtained from all the patients.

IHC assay and scoring. Tissues were fixed with $4 \%$ paraformaldehyde for at least $24 \mathrm{~h}$ at room temperature. Samples (5 $\mu \mathrm{m}$ sections) were deparaffinized in xylene and rehydrated in a graded alcohol series. Next, $3 \% \mathrm{H}_{2} \mathrm{O}_{2}$ was used to block endogenous peroxidase activity for $5 \mathrm{~min}$ at room temperature, and antigen retrieval was performed subsequent to heating in citrate buffer. The samples were blocked by normal $5 \%$ goat serum (Beijing Solarbio Science \& Technology Co., Ltd., Beijing, China) for $1 \mathrm{~h}$ at $37^{\circ} \mathrm{C}$. The sections were incubated with antibodies against Dppa4 (dilution, 1:100; Cell Signaling Technology, Inc., Danvers, MA, USA; cat. no. 67138), lactate dehydrogenase B (LDHB; dilution 1:500; Abcam, Cambridge, UK; cat. no. ab75167) and $\beta$-actin (dilution 1:5,000; Abcam; cat. no. ab8226) at $4^{\circ} \mathrm{C}$ overnight. This was followed by incubation with a horseradish peroxidase-conjugated secondary antibody (dilution, 1:2,000; GTVision III Detection kit; GeneTech Co., Ltd., Shanghai, China) at room temperature for $40 \mathrm{~min}$. The 3,3'-diaminobenzidine substrate (Sigma-Aldrich; Merck $\mathrm{KGaA}$, Darmstadt, Germany) was added for signal detection for $10 \mathrm{~min}$ at room temperature, and the sections were lightly counterstained with hematoxylin for $30 \mathrm{sec}$ at room temperature. Images were acquired using light microscopy (Olympus BX43, magnification $\mathrm{x} 400$ ).
Double blind scoring was performed independently by two investigators. Five visual fields from different areas of each specimen were randomly selected for immunohistochemical evaluation. Dppa4 expression was subjectively scored by the pathologists according to the staining intensity $(0$, no staining; 1 , weak staining; 2 , moderate staining; and 3, dark staining) and the percentage of positive cells $(0$, no positive cells; 1 , $\leq 10 \%$ positive cells; $2,10-50 \%$ positive cells; and $3,>50 \%$ positive cells). The final score was calculated as follows: Comprehensive score $=$ staining percentage $\mathrm{x}$ intensity. Dppa4 expression was defined as low in specimens with scores $<2$ and high in specimens with scores of $\geq 2$.

Cell culture. The NSCLC cell lines A549, H1299, SPC (BNCC100120) and LH7, and normal human bronchial epithelial (HBE) cells were purchased from the American Type Culture Collection (Manassas, VA, USA). A549 cells were cultured in F-12K medium (Gibco; Thermo Fisher Scientific, Inc., Waltham, MA, USA) supplemented with $10 \%$ fetal bovine serum (FBS; Gibco; Thermo Fisher Scientific, Inc.) while other cell lines were cultured in RPMI-1640 medium (Gibco; Thermo Fisher Scientific, Inc.) supplemented with 10\% FBS. All cells were incubated at $37^{\circ} \mathrm{C}$ in a humidified atmosphere with $5 \% \mathrm{CO}_{2}$. A549 cells, which had the highest Dppa4 expression, were used for Dppa4 knockdown, and H1299 cells, which had the lowest Dppa4 expression, were used for Dppa4 overexpression. The remaining cell lines were used solely for Dppa4 expression analysis in NSCLC cell lines.

Plasmids and transfection. For Dppa4 overexpression, the plasmids pcDNA3.1-Dppa4 (Dppa4-over) and control vector pcDNA3.1 were constructed as described previously (8). For expression knockdown, small interfering RNAs (siRNAs) targeting Dppa4 (namely Dppa4-si1 and Dppa4-si2; cat. no. SR310582; GenePharma Co., Ltd., Shanghai, China) were used. NSCLC cells were transfected with $4 \mu \mathrm{g}$ overexpression plasmids or $4 \mu \mathrm{g}$ siRNAs using $8 \mu \mathrm{l}$ Lipofectamine ${ }^{\circledR}$ 2000 and $8 \mu \mathrm{l}$ Lipofectamine RNAiMax (Invitrogen; Thermo Fisher Scientific, Inc.), respectively. Transfected cells were subjected to functional assays and western blotting after 24 or $48 \mathrm{~h}$, respectively. Untreated cells or cells treated with empty vectors served as the control groups. In addition, co-transfection with $4 \mu \mathrm{g}$ LDHB siRNA (cat. no. sc-45899; Santa Cruz Biotechnology, Inc., Dallas, TX, USA) and Dppa4-over plasmid in NSCLC cells was performed with Lipofectamine ${ }^{\circledR}$ 2000 reagent. The transfection efficiency was determined using western blotting $48 \mathrm{~h}$ after transfection.

Cell viability assessment. An MTT assay was used to evaluate cell viability. Briefly, the transfected $1 \times 10^{3}$ cells were plated in 96-well culture plates and cultured for 24,48 and $72 \mathrm{~h}$. Following incubation with $5 \mathrm{mg} / \mathrm{ml}$ MTT (Sigma-Aldrich; Merck KGaA) for $4 \mathrm{~h}$, the cells were washed with PBS and then solubilized with dimethyl sulfoxide. The absorbance was measured at a wavelength of $490 \mathrm{~nm}$ using a microplate reader

Western blot analysis. Standard western blotting was performed using protein $(20 \mu \mathrm{g})$ lysed by radioimmunoprecipitation assay lysis buffer (Beyotime Institute of Biotechnology, Haimen, China). The protein concentration of each group was 
subsequently determined using a bicinchoninic acid assay kit (Beyotime Institute of Biotechnology). The equal amount of proteins were separated via 10\% SDS-PAGE, and the proteins were then transferred onto polyvinylidene difluoride membranes (EMD Millipore, Billerica, MA, USA). These were subsequently blocked with $5 \%$ bovine serum albumin (BSA; Thermo Fisher Scientific, Inc.) in TBST for $1 \mathrm{~h}$ at room temperature. The samples were incubated with primary antibodies against Dppa4 (dilution, 1:1,000; Cell Signaling Technology, Inc.; cat. no. 67138), LDHB (dilution 1:1,000; Abcam; cat. no. ab75167) and $\beta$-actin (dilution 1:5,000; Abcam; cat. no. ab8226) overnight at $4^{\circ} \mathrm{C}$. TBST was used to wash the PVDF membranes three times, which were then incubated with a secondary antibody anti-mouse (dilution, 1:1,000; Cell Signaling Technology, Inc.; cat. no. 7076) and anti-rabbit (dilution, 1:1,000; Cell Signaling Technology, Inc.; cat. no.7074) for $1 \mathrm{~h}$ in the room temperature. TBST was used to wash again three times. Finally, proteins were visualized using an enhanced chemiluminescence kit (Thermo Fisher Scientific, Inc.), and images were acquired using ImageQuant LAS 4000 (GE Healthcare Life Sciences, Little Chalfont, UK) and densitometry analysis was performed using ImageQuant TL software version 1.1 (GE Healthcare Life Sciences).

RNA isolation and reverse transcription-quantitative polymerase chain reaction $(R T-q P C R)$ analysis. NSCLC cell lines and tumor samples were used to detect the Dppa4 expression by RT-qPCR. Furthermore, the relative levels of glycolytic enzyme expression, namely GLUT-1, GLUT-4, phosphofructokinase liver type (PFK-L), hexokinase 2 (HK-II), phosphofructokinase muscle type (PFK-M), pyruvate kinase M-1 (PKM-1), PKM-2, phosphofructokinase platelet (PFK-P), aldolase, fructose-bisphosphate B (AldoB), phosphoglycerate kinase 1 (PGK-1), phosphoglycerate mutase (PGAM-1), Enolase, glucose-6-phosphate isomerase (G6PI), LDHA and LDHB. Total RNA extraction was performed using TRIzol ${ }^{\circledR}$ reagent (Thermo Fisher Scientific, Inc.) for cell cultures and the miniBEST universal RNA extraction kit for fresh tumor and tumor-adjacent tissues (Takara Bio, Inc., Otsu, Japan). RNA was then reverse transcribed to cDNA (PrimeScript ${ }^{\mathrm{TM}}$ RT reagent Kit with genomic DNA Eraser; Takara Bio, Inc.), which was then subjected to qPCR analysis (SYBR Green PCR kit, Takara Bio, Inc.) to evaluate mRNA expression. The RT temperature protocol was: $37^{\circ} \mathrm{C}$ for $15 \mathrm{~min}$ and $85^{\circ} \mathrm{C}$ for $5 \mathrm{sec}$. PCR amplification was performed in a thermal cycler for 40 cycles at the following cycle conditions: $95^{\circ} \mathrm{C}$ for $5 \mathrm{sec}, 60^{\circ} \mathrm{C}$ for $30 \mathrm{sec}$, and $72^{\circ} \mathrm{C}$ for $30 \mathrm{sec}$. The relative mRNA expression of each gene was calculated with the comparative quantitation cycle $(\mathrm{Cq})$ method, $2^{-\Delta \Delta \mathrm{Cq}}(9,10)$. The specific primers used in qPCR were as follows: Dppa4 sense, 5'-GACACAGATGGTTGGGTT CA-3', and antisense, 5'-GAGGCAGGAAGCAAGAAGAG-3'; GAPDH sense, 5'-AGAAGGCTGGGGCTCATTTG-3', and antisense, 5'-AGGGGCCATCCACAGTCTTC-3'; GLUT-1 sense, 5'-CTTTGTGGCCTTCTTTGAAGT-3', and anti-sense, 5'-CCACACAGTTGCTCCACAT-3'; GLUT-4 sense, 5'-CTT CATCATTGGCATGGGTTT-3', and antisense, 5'-CGGGTT TCAGGCACTTTTAGG-3'; PFK-L sense, 5'-GGACAGGAA AGAGGAAGTGAC-3', and antisense, 5'-CGTAGATGAGGA AGACTTTGGC-3'; HK-II sense, 5'-GATTTCACCAAGCGT GGACT-3', and antisense, 5'-CCACACCCACTGTCACTT
TG-3'; PFK-M sense, 5'-ATTCGGGCTGTGTTCTGG-3', and antisense, 5'-TGGCTAGGATTTTGAGGATGG-3'; PKM-1 sense, 5'-CTATCCTCTGGAGGCTGTGC-3', and antisense, 5'-CCATGAGGTCTGTGGAGTGA-3'; PKM-2 sense, 5'-GGGTTCGGAGGTTTGATG-3', and antisense, 5'-ACG GCGGTGGCTTCTGT-3'; PFK-P sense, 5'-CATCGACAA TGATTTCTGCGG-3', and antisense, 5'-CCATCACCTCCA GAACGAAG-3'; AldoB sense, 5'-ATGCCACTCTCAACC TCAATGCTATC-3', and antisense, 5'-TTATTTTCTTGG GTGGGTATTCTGG-3'; PGK-1 sense, 5'-CGGTAGTCC TTATGAGCC-3', and antisense, 5'-CATGAAAGCGGAGGT TCT-3'; PGAM-1 sense, 5'-CCTGGAGAACCGCTTC-3', and antisense, 5'-CATGGGCTGCAATCAGTACAC-3'; Enolase sense, 5'-CTGATGCTGGAGTTGGATGG-3', and antisense, 5'-CCATTGATCACGTTGAAGGC-3'; G6PI sense, 5'-AGG CTGCTGCCACATAAGGT-3', and antisense, 5'-AGCGTC GTGAGAGGTCACTTG-3'; LDHA sense, 5'-CAGCTTGGA GTTTGCAGTTAC-3', and antisense, 5'-TGATGGATCTCC AACATGG-3'; LDHB sense, 5'-CCTAGAGCTCACTAGTCA CAG-3' and antisense, 5'-CTCCTGTGCAAAATGGCAAC-3'.

Lactate dehydrogenase $(\mathrm{LDH})$ activity, lactate production, glucose utilization assay and intracellular ATP level. For $\mathrm{LDH}$ activity and lactate production assays, tumor cells were transfected with siRNAs and plasmids, and after $24 \mathrm{~h}$ a total of $1 \times 10^{6}$ cells were examined using the Lactate Dehydrogenase Activity Assay kit and Lactate Assay kit (Sigma-Aldrich; Merck KGaA) according to the manufacturer's protocol. For the glucose utilization assay, tumor cells were transfected with plasmids and siRNAs for $24 \mathrm{~h}$, and then the media were replaced with phenol red-free RPMI medium supplemented with $1 \%$ FBS, followed by continuous culture for 3 days. The glucose utilization was measured using a colorimetric glucose assay kit (BioVision Inc., Milpitas, CA, USA) and normalized to the cell number. For intracellular ATP measurement, a firefly luciferase-based ATP assay kit (Beyotime Institute of Biotechnology) was used, according to the manufacturer's instructions.

Statistical analysis. All data are presented as the mean \pm standard deviation. The comparisons between tumor tissue samples and control tissues were performed using a paired t-test. The mean values of other two groups were compared with the Student's t-test, while the mean values of three or more groups were compared by one-way analysis of variance. Statistical analyses were conducted with SPSS for Windows, version 17.0 (SPSS, Inc., Chicago, IL, USA). The least-significant difference test was used in the comparison of two pairs as a post hoc test. Associations between the expression of Dppa4 and clinical characteristics were analyzed with $\chi^{2}$ test or Fisher's exact test, as appropriate. The overall survival was assessed using the Kaplan-Meier method. Univariate and multivariate Cox regression analyses were also performed. Parameters with a P-value of $<0.05$ in the univariate analysis were included in a Cox multivariate proportional hazards regression model. In the multivariate analysis, the Backward LR statistic was used in the conditional logistic regression model. All statistical analyses were performed using SPSS version 17.0 software. $\mathrm{P}<0.05$ was considered to indicate a statistically significant difference. 
Table I. Comparison of Dppa4 expression between NSCLC and paired adjacent normal tissues.

\begin{tabular}{lcccc}
\hline & & \multicolumn{2}{c}{ Dppa4 expression } & \\
\cline { 3 - 4 } Tissues & Cases & Low (\%) & High (\%) & P-value \\
\hline NSCLC & 80 & $38(47.5)$ & $42(52.5)$ & $<0.001^{\text {a }}$ \\
Adjacent normal & 80 & $65(81.3)$ & $15(18.7)$ & \\
\hline
\end{tabular}

${ }^{\mathrm{a}} \mathrm{P}<0.05$. Dppa4, developmental pluripotency-associated 4; NSCLC, non-small cell lung carcinoma.

\section{Results}

Aberrant overexpression of Dppa4 in NSCLC tissues and cells. The mRNA expression of Dppa4 was evaluated in 20 pairs of NSCLC tissues and adjacent normal tissues, while a total of 80 paired tissue samples were used to examine the Dppa4 protein level. The results demonstrated that the expression of Dppa4 was higher in NSCLC tissue samples as compared with that in normal tissues, with a statistically significant difference detected $(\mathrm{P}<0.05$; Fig. $1 \mathrm{~A}$ and Table I). Representative images of Dppa4 expression in adenocarcinoma and squamous cell carcinoma are shown in Fig. 1B. Subsequently, these results were verified in NSCLC cell lines. Compared with the normal HBE cells, the tumor A549, SPC and LH7 cell lines exhibited markedly higher expression of Dppa4 at both the mRNA and protein levels, with no significant difference detected in H1299 cells $(\mathrm{P}<0.05$; Fig. $1 \mathrm{C}$ and $\mathrm{D})$.

Correlation between Dppa4 expression and the clinicopathological characteristics of NSCLC patients. The correlation between Dppa4 expression and the clinicopathological characteristics of NSCLC patients was next investigated. As shown in Table II, Dppa4 was positively correlated with the tumor differentiation grade $(\mathrm{P}=0.04)$, $\mathrm{T}$ stage $(\mathrm{P}=0.006), \mathrm{N}$ stage $(\mathrm{P}<0.001)$ and clinical stage $(\mathrm{P}<0.001)$. Other clinicopathological characteristics, including the patient sex, age and smoking history, were not found to be significantly correlated with Dppa4 expression. These data suggest that Dppa4 may be involved in NSCLC progression.

High Dppa4 expression is associated with poor clinical outcomes in NSCLC patients. To further explore the association between Dppa4 expression and patient prognosis, a Kaplan-Meier analysis was conducted. As shown in Fig. 1E, the analysis revealed that patients with higher Dppa4 expression exhibited a poorer prognosis. As shown in Table III, higher Dppa4 levels were significantly associated with lower 1-, 3- and 5-year survival rates (Table III). Furthermore, univariate survival analysis indicated that, high Dppa4 expression predicted poor prognosis in advanced TNM stage of IIIA ( $\mathrm{P}<0.05$; Table IV). Subsequent multivariate analysis revealed that high Dppa4 expression [hazard ratio (HR), 1.862; 95\% confidence interval (CI), 1.090-3.181; $\mathrm{P}=0.023$; Table IV] and TNM stage of IIIA (HR=2.063; 95\% CI, 1.148-3.708; $\mathrm{P}=0.015$; Table IV) were correlated with overall survival. Taken together, these findings demonstrated that
Table II. Correlation between the clinicopathological characteristics and Dppa4 expression in non-small cell lung carcinoma patients.

\begin{tabular}{|c|c|c|c|c|}
\hline \multirow[b]{2}{*}{ Characteristics } & \multirow[b]{2}{*}{$\mathrm{N}$} & \multicolumn{2}{|c|}{$\begin{array}{c}\text { Dppa4 } \\
\text { expression }\end{array}$} & \multirow[b]{2}{*}{ P-value } \\
\hline & & $\begin{array}{l}\text { Low } \\
(\mathrm{n}=38)\end{array}$ & $\begin{array}{l}\text { High } \\
(\mathrm{n}=42)\end{array}$ & \\
\hline Age (years) & & & & 0.479 \\
\hline$\leq 60$ & 37 & 16 & 21 & \\
\hline$>60$ & 43 & 22 & 21 & \\
\hline Sex & & & & 0.538 \\
\hline Male & 49 & 24 & 25 & \\
\hline Female & 31 & 13 & 18 & \\
\hline Smoking history & & & & 0.685 \\
\hline No & 37 & 24 & 13 & \\
\hline Yes & 43 & 26 & 17 & \\
\hline Histological type & & & & 0.220 \\
\hline Adenocarcinoma & 32 & 17 & 15 & \\
\hline Squamous cell carcinoma & 32 & 11 & 21 & \\
\hline Other & 16 & 9 & 7 & \\
\hline Grade & & & & $0.04^{\mathrm{a}}$ \\
\hline Well-differentiated & 51 & 28 & 23 & \\
\hline $\begin{array}{l}\text { Moderate/Poor } \\
\text { differentiation }\end{array}$ & 29 & 9 & 20 & \\
\hline T stage & & & & $0.006^{\mathrm{a}}$ \\
\hline $\mathrm{T} 1$ & 23 & 11 & 12 & \\
\hline $\mathrm{T} 2 \mathrm{a}$ & 40 & 24 & 16 & \\
\hline $\mathrm{T} 2 \mathrm{~b}$ & 16 & 2 & 14 & \\
\hline $\mathrm{T} 3$ & 1 & 0 & 1 & \\
\hline $\mathrm{N}$ stage & & & & $<0.001^{\mathrm{a}}$ \\
\hline No & 20 & 15 & 5 & \\
\hline N1 & 33 & 19 & 14 & \\
\hline $\mathrm{N} 2$ & 27 & 3 & 24 & \\
\hline TNM stage & & & & $<0.001^{\mathrm{a}}$ \\
\hline $\mathrm{I}+\mathrm{II}$ & 53 & 34 & 19 & \\
\hline IIIA & 27 & 3 & 24 & \\
\hline
\end{tabular}

${ }^{\mathrm{a}} \mathrm{P}<0.05$

Dppa4 expression may be an independent prognostic marker for NSCLC patients.

Dppa4 knockdown decreases NSCLC cell viability and glycolysis. To determine the effect of altered Dppa4 expression on NSCLC biology, siRNA transfection was performed in A549 cells. As shown in Fig. 2A, Dppa4 expression was significantly decreased in A549 cells transfected with Dppa4-si1 and Dppa4-si2 compared with the control group, verifying the efficacy of Dppa4 knockdown. Next, the biological effects of Dppa4 knockdown on NSCLC were evaluated. It was observed that Dppa4 downregulation significantly inhibited cell viability (Fig. $2 \mathrm{~B} ; \mathrm{P}<0.05$ ). 
Table III. Comparison of cumulative survival rate between the low and high Dppa4 expression groups.

\begin{tabular}{lcrr}
\hline & & \multicolumn{2}{c}{ Cumulative survival rate, 95\% confidence interval } \\
\cline { 2 - 4 } Dppa4 expression & 1-year & 3-year & 5 -year \\
\hline Low & $86 \%(0.742-0.978)$ & $40 \%(0.224-0.576)$ & $7 \%(-0.028-0.168)$ \\
High & $48 \%(0.323-0.637)$ & $13 \%(0.012-0.248)$ & $2 \%(-0.039-0.079)$ \\
\hline
\end{tabular}

A
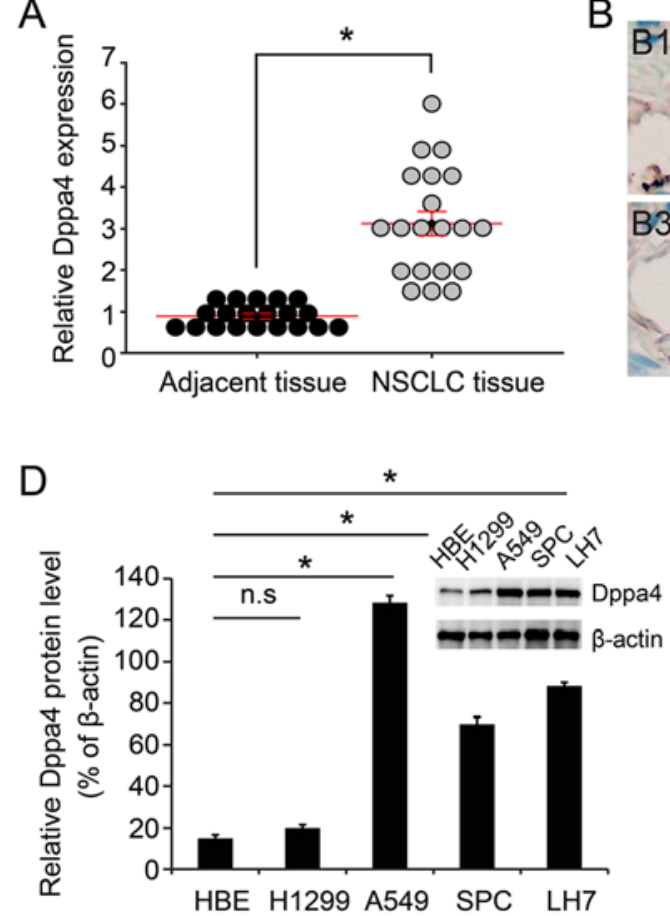

$\mathrm{B}$

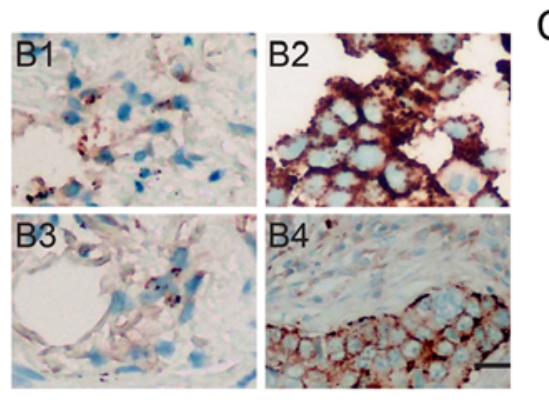

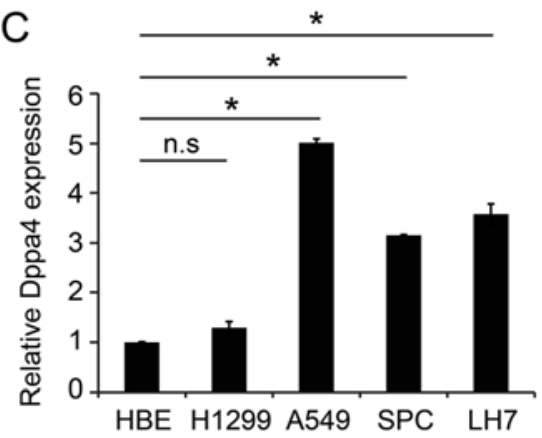

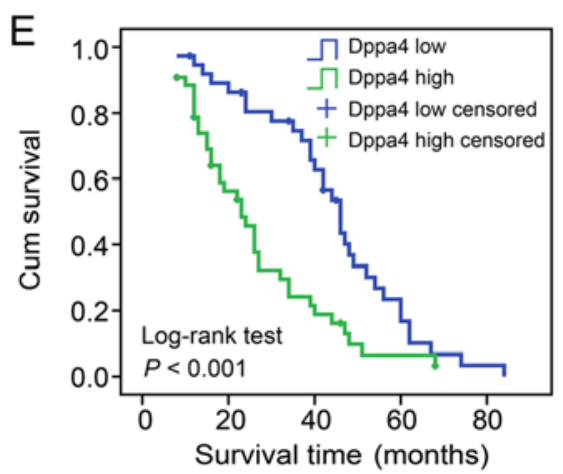

Figure 1. Dppa4 overexpression in NSCLC is correlated with worse survival. (A) RT-qPCR demonstrated higher mRNA expression of Dppa4 in NSCLC tissues compared with that in adjacent normal tissues. (B) Representative images of Dppa4 expression in adenocarcinoma (B1, adjacent normal tissues; B2, tumor tissues) and in squamous cell carcinoma (B3, adjacent normal tissues; B4, tumor tissues). (C) RT-qPCR and (D) western blotting respectively demonstrated higher mRNA and expression levels of Dppa4 in NSCLC cell lines compared with those in normal HBE cells. (E) Kaplan-Meier analysis revealed that patients with higher Dppa4 expression exhibited poorer prognosis. ${ }^{*} \mathrm{P}<0.05$. Dppa4, developmental pluripotency-associated 4; NSCLC, non-small-cell lung cancer; RT-qPCR, reverse transcription-quantitative polymerase chain reaction; HBE, human bronchial epithelial; n.s., not significant.

As altered aerobic glycolysis is a characteristic of cancer metabolism, the present study then focused on the effect of Dppa4 regulation of glycolysis on NSCLC metabolism. Following Dppa4 knockdown, significant decreases in glucose utilization, lactate production and LDH activity were observed, along with a marked increase in the intracellular ATP level $(\mathrm{P}<0.05$; Fig. 2C), suggesting a decrease in glycolysis.

Dppa4 overexpression increases cell proliferation and glycolysis. Overexpression of Dppa4 was subsequently induced in H1299 cells. As shown in Fig. 3A, Dppa4 expression was increased in H1299 cells transfected with the Dppa4-over plasmid, therefore verifying that overexpression was successfully induced. It was then observed that Dppa4 overexpression significantly promoted cell growth $(\mathrm{P}<0.05$; Fig. 3B). Furthermore, the Dppa4 upregulation was associated with significant increases in glucose utilization, lactate production and LDH activity, along with a decrease in the intracellular ATP level ( $\mathrm{P}<0.05$; Fig. 3C).
Dppa4 regulates the levels of glycolytic enzymes in NSCLC cells. The effect of Dppa4 on the expression levels of glycolytic enzymes in NSCLC cells was then screened. It was observed that knockdown of Dppa4 expression had no evident effect on the majority of enzymes examined; however, significant upregulation of glucose transporter type 4 (GLUT-4) and pyruvate kinase isozyme M2 (PKM2), and marked downregulation of hexokinase II (HK-II) and LDHB ( $\mathrm{P}<0.05$; Fig. 4A) were observed in the Dppa4 knockdown groups. In addition, Dppa4 overexpression had no marked effect on the majority of the enzymes, with the exception of GLUT-4 and LDHA downregulation, and the upregulation of HK-II, enolase and LDHB $(\mathrm{P}<0.05$; Fig. 4B). Among the abovementioned enzymes, LDHB exhibited the most prominent changes.

$L D H B$ reverses the promoting effects of Dppa4 in NSCLC cells. To elucidate the importance of LDHB expression in NSCLC progression, Dppa4 overexpression/control plasmid and LDHB/control siRNA were co-transfected in the cells 
Table IV. Summary of univariate and multivariate Cox regression analyses of overall survival duration in all non-small cell lung carcinoma patients.

\begin{tabular}{|c|c|c|c|c|c|c|}
\hline \multirow[b]{2}{*}{ Characteristic } & \multicolumn{3}{|c|}{ Univariate analysis } & \multicolumn{3}{|c|}{ Multivariate analysis } \\
\hline & HR & $95 \% \mathrm{CI}$ & P-value & $\mathrm{HR}$ & $95 \% \mathrm{CI}$ & P-value \\
\hline \multicolumn{7}{|l|}{ Dppa4 expression } \\
\hline Low & 1 & & & 1 & & \\
\hline High & 2.271 & $1.384-3.727$ & $<0.001^{\mathrm{a}}$ & 1.862 & $1.090-3.181$ & $0.023^{\mathrm{a}}$ \\
\hline \multicolumn{7}{|l|}{ Age(years) } \\
\hline$\leq 60$ & 1 & & & & & \\
\hline$>60$ & 0.777 & $0.479-1.260$ & 0.306 & & & \\
\hline \multicolumn{7}{|l|}{ Sex } \\
\hline Male & 1 & & & & & \\
\hline Female & 1.086 & $0.658-1.793$ & 0.747 & & & \\
\hline \multicolumn{7}{|l|}{ Smoking history } \\
\hline Never & 1 & & & & & \\
\hline Ever & 0.795 & $0.483-1.308$ & 0.366 & & & \\
\hline \multicolumn{7}{|l|}{ Histological type } \\
\hline Adenocarcinoma & 1 & & & & & \\
\hline Squamous cell carcinoma & 0.951 & $0.552-1.638$ & 0.857 & & & \\
\hline Others & 1.009 & $0.516-1.972$ & 0.980 & & & \\
\hline \multicolumn{7}{|l|}{ Grade } \\
\hline Well-differentiated & 1 & & & & & \\
\hline Moderate/Poor differentiation & 1.363 & $0.835-2.224$ & 0.216 & & & \\
\hline \multicolumn{7}{|l|}{ T stage } \\
\hline $\mathrm{T} 1$ & 1 & & & & & \\
\hline $\mathrm{T} 2 \mathrm{a}$ & 0.731 & $0.416-1.284$ & 0.275 & & & \\
\hline $\mathrm{T} 2 \mathrm{~b}$ & 2.004 & $1.013-3.964$ & $0.046^{\mathrm{a}}$ & & & \\
\hline $\mathrm{T} 3$ & 7.885 & $0.973-63.877$ & 0.053 & & & \\
\hline \multicolumn{7}{|l|}{$\mathrm{N}$ stage } \\
\hline N0 & 1 & & & & & \\
\hline $\mathrm{N} 1$ & 2.225 & $1.233-4.015$ & $0.008^{\mathrm{a}}$ & & & \\
\hline $\mathrm{N} 2$ & 0.691 & $0.380-1.257$ & 0.226 & & & \\
\hline \multicolumn{7}{|l|}{ TNM stage } \\
\hline $\mathrm{I}+\mathrm{II}$ & 1 & & & 1 & & \\
\hline IIIA & 2.616 & $1.520-4.502$ & $<0.001^{\mathrm{a}}$ & 2.063 & $1.148-3.708$ & $0.015^{\mathrm{a}}$ \\
\hline
\end{tabular}

${ }^{\mathrm{a}} \mathrm{P}<0.05$. HR, hazard ratio; $95 \% \mathrm{CI}, 95 \%$ confidence interval.

(Fig. 5A). The results demonstrated that LDHB siRNA reversed the promoting effect of Dppa4 overexpression on cell proliferation (Fig. 5B) and glycolysis (Fig. 5C). These findings indicated that Dppa4 promotes NSCLC progression and this effect is partly mediated via LDHB.

\section{Discussion}

Dppa4, a protein that is located in the embryonic stem cell nucleus and is associated with chromatin, has been reported to regulate the differentiation of ES cells into a primitive ectodermal lineage (11). Although the expression of Dppa4 in ES cells has been extensively investigated, little is known regarding its in vivo expression in cancer. Previous research has indicated that human cancer may be considered as a stem-cell disease (12). Cancer-initiating cells, also referred to as cancer stem cells, serve an important role in hematopoietic cancer, as well as in several solid tumors, such as lung cancer (13).

The aim of the present study was to investigate the role of Dppa4 in NSCLC tumorigenesis. Initially, Dppa4 was found to be highly expressed in NSCLC tissue samples and cell lines, both at the mRNA and protein levels. Next, the correlation between Dppa4 expression and clinicopathological characteristics in NSCLC patients was assessed. It was observed that Dppa4 was positively correlated with tumor differentiation grade, $\mathrm{T}$ stage, $\mathrm{N}$ stage and clinical stage. Subsequent Kaplan-Meier analysis revealed that patients with higher Dppa4 expression exhibited poorer prognosis. Univariate and multivariate survival analyses 

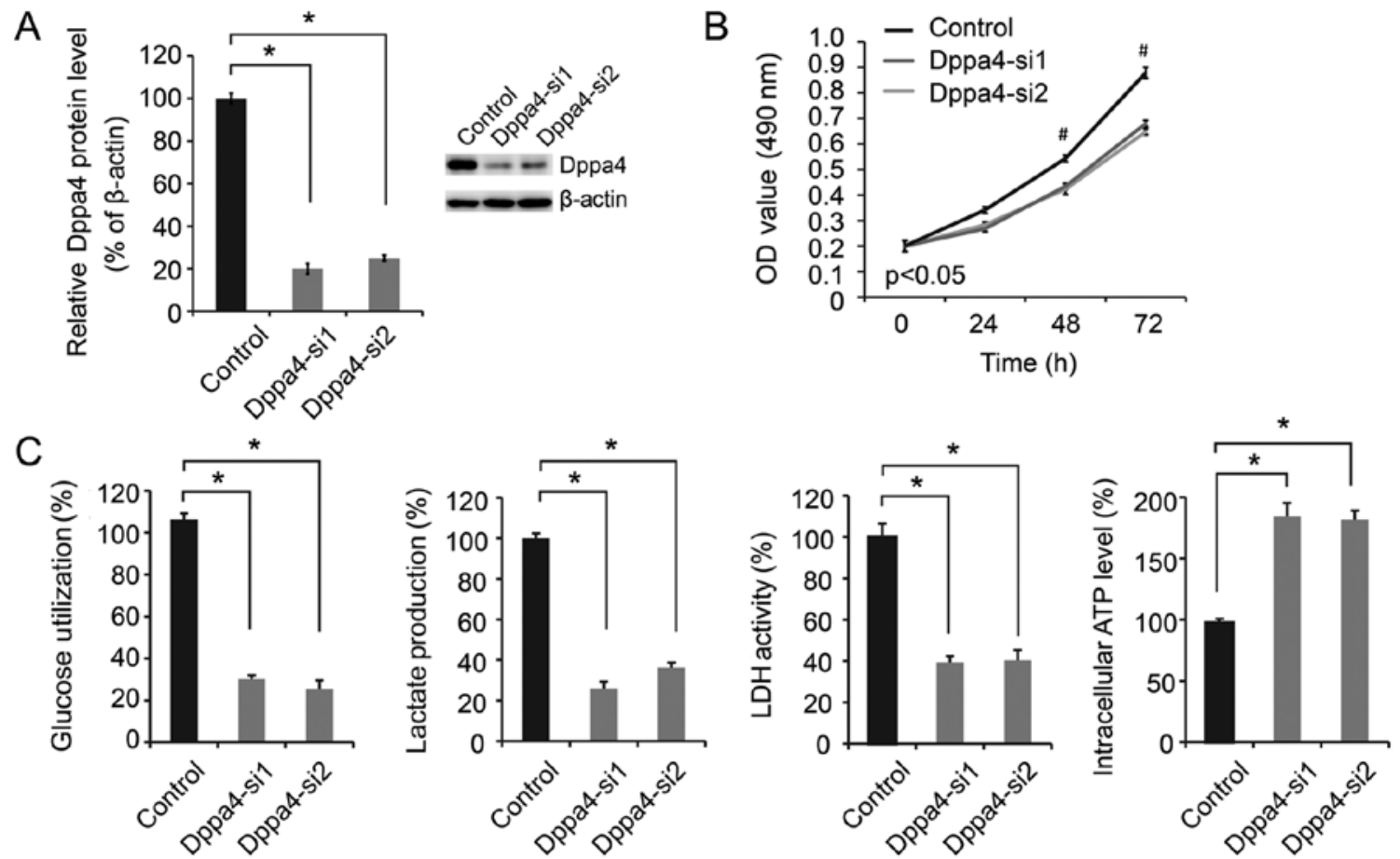

Figure 2. Dppa4 knockdown decreased cancer cell proliferation and glycolysis. (A) Western blotting subsequent to siRNA transfection (Dppa4-si1 and Dppa4-si2) revealed decreased Dppa4 expression in A549 cells. (B) Dppa4 knockdown markedly inhibited cell growth. (C) Significant decreases in glucose use, lactate production and LDH activity, along with an increase in the intracellular ATP level were observed following Dppa4 knockdown, also known as the Warburg effect. "P<0.05; " $\mathrm{P}<0.05$ vs. Dppa4-si1 and Dppa4-si2 groups. Dppa4, developmental pluripotency-associated 4; LDH, lactate dehydrogenase; siRNA, small interfering RNA; OD optical density.

A

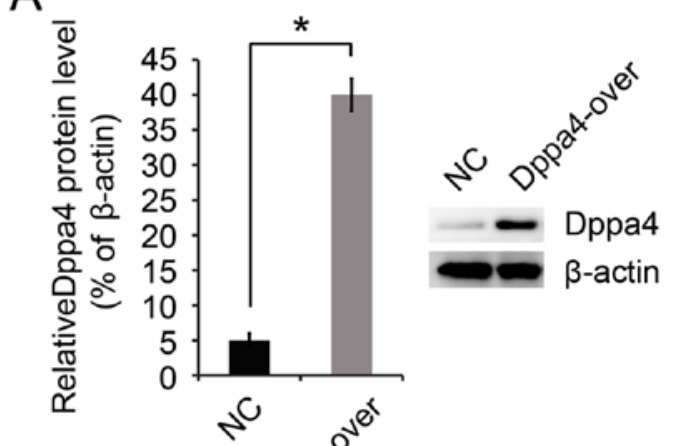

B
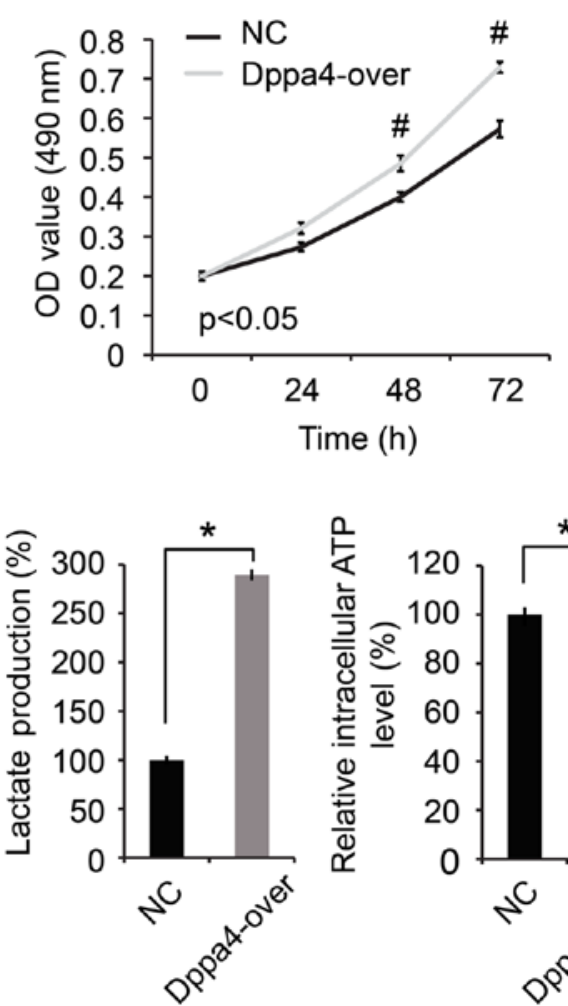

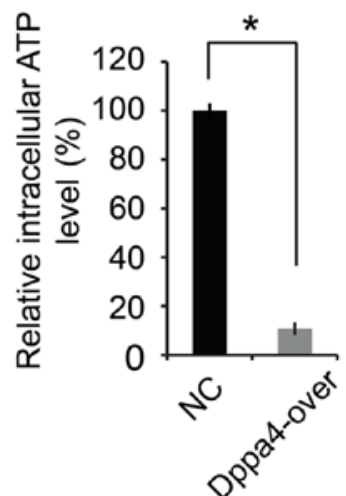

Figure 3. Dppa4 overexpression increased cancer cell proliferation and glycolysis. (A) Western blotting subsequent to the introduction of a Dppa4 overexpression plasmid revealed increased Dppa4 expression in H1299 cells. (B) Dppa4 overexpression significantly promoted cell growth. (C) Significant increases in glucose utilization, lactate production and LDH activity, along with a decrease in the intracellular ATP level, were observed following Dppa4 knockdown, also known as the Warburg effect. ${ }^{*} \mathrm{P}<0.05 ;{ }^{\#} \mathrm{P}<0.05$ vs. NC group. Dppa4, developmental pluripotency-associated 4; LDH, lactate dehydrogenase; NC, negative control. 

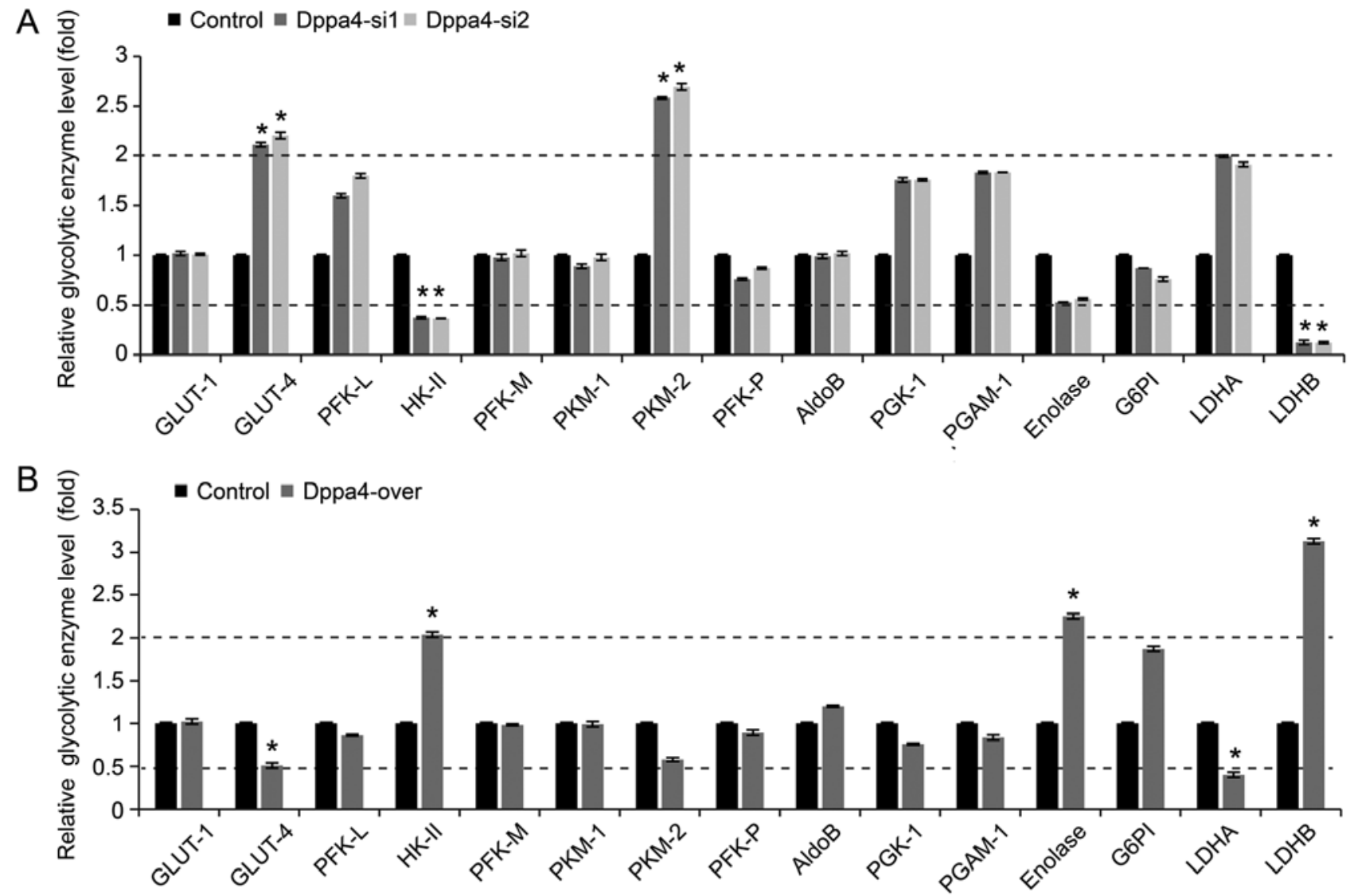

Figure 4. Expression of glycolysis-associated enzymes in non-small-cell lung cancer cells. (A) Knockdown of Dppa4 expression did not alter the expression of the majority of glycolysis-associated enzymes, with the exception of GLUT-4 and PKM2 upregulation, and HK-II and LDHB downregulation. (B) Dppa4 overexpression did not affect the expression of the majority of these enzymes, apart from the downregulation of GLUT-4 and LDHA, and the upregulation of HK-II, enolase and LDHB. "P<0.05 vs. corresponding control group. Dppa4, developmental pluripotency-associated 4; GLUT-4, glucose transporter type 4; PKM2, pyruvate kinase isozyme M2; HK-II, hexokinase II; LDHB, lactate dehydrogenase B.
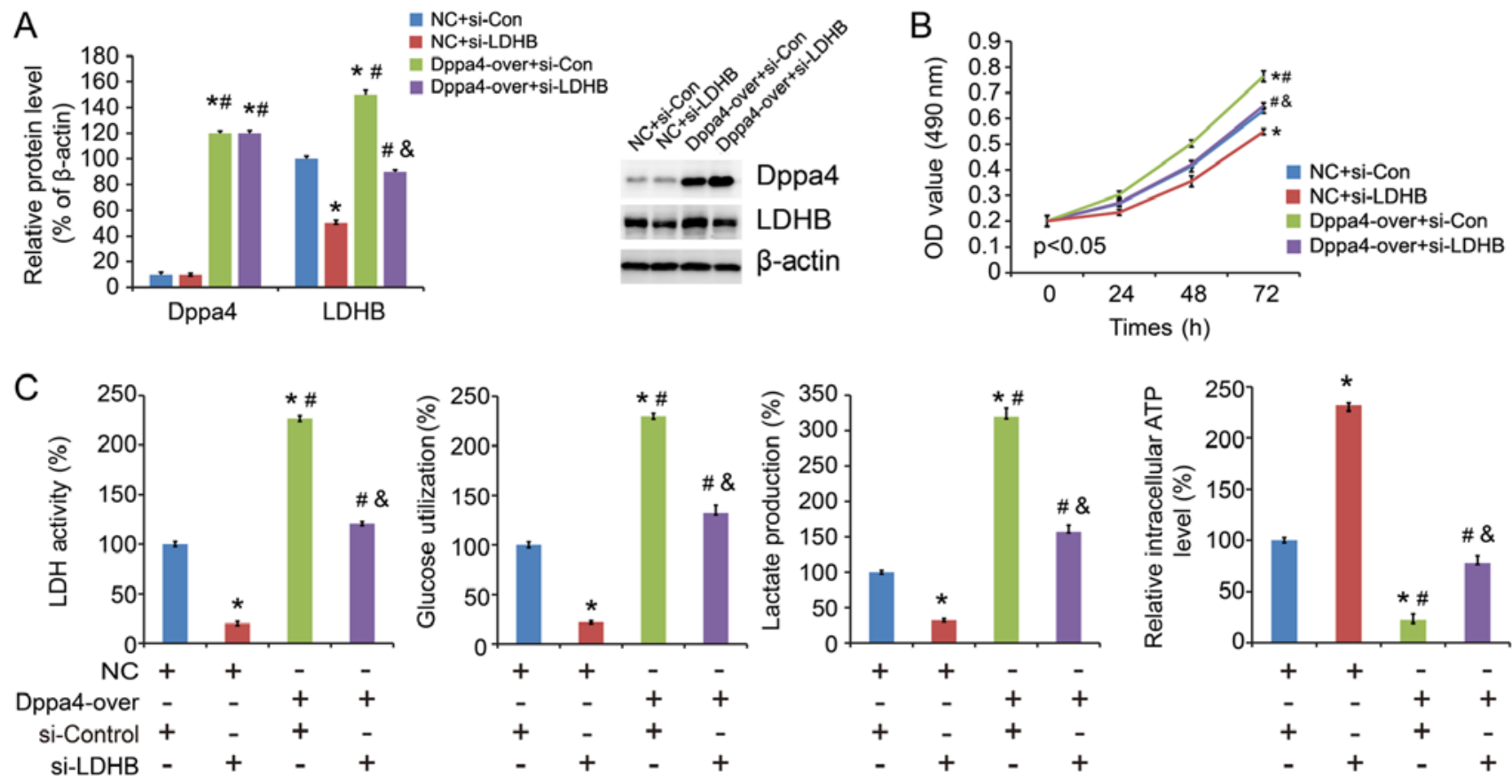

Figure 5. LDHB reversed the promoting effects of Dppa4 in non-small-cell lung cancer cells. (A) Verification of protein expression changes following co-transfection of cells with Dppa4-over/NC plasmid and si-LDHB/si-Con. (B) Cell proliferation and (C) glycolysis of cells transfected with Dppa4-over/NC plasmid or si-LDHB/si-Con. LDHB downregulation reversed the promoting effect of Dppa4 overexpression on cancer cell proliferation and glycolysis. ${ }^{\text {"P }}<0.05$ vs. control group; ${ }^{\mathrm{P}} \mathrm{P}<0.05$ vs. NC+si-LDHB group; ${ }^{\circledR} \mathrm{P}<0.05$ vs. Dppa4-over+si-Con group. LDHB, lactate dehydrogenase B; Dppa4, developmental pluripotency-associated 4; Dppa4-over, Dppa4 overexpression; NC, negative control; si-, small interfering RNA; Con, control; OD, optical density. 
also revealed that, in addition to tumor stage, upregulated Dppa4 expression was a predictor of poor prognosis. However, the underlying mechanism remained unclear.

The key role of Dppa4 in NSCLC development and progression was demonstrated in the present study; however, the currently available literature contains little evidence regarding the role of Dppa4 in cancer metabolism. Abnormal metabolism is regarded as one of the characteristic features of cancer. Since the energy produced by glycolysis may contribute to the abnormal proliferation of cancer cells, the current study focused on whether glycolysis affected cell proliferation. It is widely accepted that cancer growth depends on metabolism, and its metabolic pattern shifts from aerobic to anaerobic respiration. Under the condition of sufficient oxygen, the glycolysis of malignant tumor cells is also active. The metabolic characteristic of this aerobic glycolysis is known as the Warburg effect, which displays high glucose uptake rate, active glycolysis and high lactic acid content of metabolites. Furthermore, Jones et al (14) reported that cancer stem cells have a third metabolic mode. On the basis of maintaining the aerobic mode, glucose metabolism is converted into protein catabolism, which provides energy for tumor cells (14). Malignant tumor cells perform glycolysis at a rate that is 10 times faster than that observed in their non-cancerous counterparts (15). To generate energy, cancer cells convert glucose into lactate, supplying a primary route for the carbon source that is required for macromolecular biosynthesis. This process enables cancer cells to meet the increasing energy demands of rapid tumor growth $(16,17)$.

In the present study, Dppa4 knockdown had no evident effect on the expression of the majority of the glycolysis-associated enzymes, with the exception of upregulation of GLUT-4 and PKM2, and downregulation of HK-II and LDHB. Similarly, Dppa4 overexpression exerted no marked effect on the levels of the majority of these enzymes, apart from downregulation of GLUT-4 and LDHA, and upregulation of HK-II, enolase and LDHB. The most notable changes as a result of Dppa4 knockdown or overexpression were reported in LDHB. Among the abovementioned enzymes, PKM1 and PKM2 are alternative splicing products of the PKM gene, which have distinct functions and expression characteristics (18). Only PKM2 is expressed in embryonic tissues, whereas the PKM1 type is expressed during adulthood, however, PKM2 expression is observed in tumor tissues, rather than PKM1 (18). During tumor formation, PKM1 or PKML/R gradually disappear, while PKM2 is markedly upregulated, ultimately becoming a tumor-specific pyruvate kinase (18). In addition, HK-II has been reported to accelerate glucose metabolism and promote cancer progression (19). Furthermore, insulin resistance is known to serve an important role in tumor cachexia. A study by Yoshikawa et al (20) demonstrated that insulin resistance in tumor-tolerant mice was due to a decrease in the expression of GLUT-4.

LDH, comprising LDHA and LDHB, is a terminal enzyme catalyzing the interconversion of pyruvate and lactate in the anaerobic glycolytic pathway, and is considered as the key glycolytic enzyme $(21,22)$. With regard to LDHB expression, it differs markedly among different malignant tumors. Certain studies have reported that the high expression of LDHB in lung and breast cancer enhances the proliferation of tumor cells, and is a significant predictor of poor prognosis (23-25). Other studies have demonstrated that LDHB expression is suppressed in hepatocellular carcinoma (HCC) (26), and prostate (21), pancreatic (27) and gastric cancer (28). Furthermore, LDHB may act as a suppressor of glycolysis, thereby suppressing pancreatic cancer and HCC progression. The mechanism may involve promoter hypermethylation and decreased expression of LDHB, leading to glycolytic transition by converting lactate to pyruvate, and a shift from LDH1 to LDH5 (29). As a therapeutic target, LDHB should be approached differently in different types of cancer. Investigating the importance of LDHB in NSCLC progression revealed that transfection with LDHB siRNA reversed the promoting effect of Dppa4 overexpression on cancer cell proliferation and glycolysis. These findings indicate that Dppa4 promotes NSCLC cell proliferation and glycolysis in part via LDHB.

In conclusion, the present study, to the best of our knowledge, is the first to provide critical insight into the role of Dppa4 in NSCLC cell proliferation and glycolysis, and to indicate a role for Dppa4-LDHB signaling in NSCLC development and progression. The findings not only indicated a newly identified molecular mechanism involved in NSCLC glycolysis and progression, but also highlighted the Dppa4-LDHB axis as a novel promising molecular target for the design of new therapeutic strategies for NSCLC.

\section{Acknowledgements}

Not applicable.

\section{Funding}

No funding was received.

\section{Availability of data and materials}

The datasets generated and analyzed in the present study are available from the corresponding author on reasonable request.

\section{Authors' contributions}

LL and YFW performed the cellular and histological studies, and statistical analysis, and drafted the manuscript. QW and FS collected tumor tissues and follow up information on the patients. JQ, XW, JX and YJW assisted in performing the cellular and histological studies. YZ participated in the study design. All authors have read and approved the final version of the manuscript.

\section{Ethics approval and consent to participate}

The study protocol was approved by the Research Ethics Committee of Xuzhou Cancer Hospital (Xuzhou, China). Informed consent was obtained from all the patients.

\section{Patient consent for publication}

Not applicable.

\section{Competing interests}

The authors declare that they have no competing interests. 


\section{References}

1. Siegel RL, Miller KD and Jemal A: Cancer statistics, 2018. CA Cancer J Clin 68: 7-30, 2018.

2. Ezeh UI, Turek PJ, Reijo RA and Clark AT: Human embryonic stem cell genes OCT4, NANOG, STELLAR, and GDF3 are expressed in both seminoma and breast carcinoma. Cancer 104: 2255-2265, 2005.

3. Pardal R, Clarke MF and Morrison SJ: Applying the principles of stem-cell biology to cancer. Nat Rev Cancer 3: 895-902, 2003.

4. Amini S, Fathi F, Mobalegi J, Sofimajidpour H and Ghadimi T: The expressions of stem cell markers: Oct4, Nanog, Sox2, nucleostemin, Bmi, Zfx, Tcl1, Tbx3, Dppa4, and Esrrb in bladder colon, and prostate cancer, and certain cancer cell lines. Anat Cell Biol 47: 1-11, 2014

5. Du J, Chen T, Zou X, Xiong B and Lu G: Dppa2 knockdown-induced differentiation and repressed proliferation of mouse embryonic stem cells. J Biochem 147: 265-271, 2010.

6. Zhang M, Cui F, Lu S, Lu H, Xue Y, Wang J, Chen J, Zhao S, Ma S, Zhang Y, et al: Developmental pluripotency-associated 4: A novel predictor for prognosis and a potential therapeutic target for colon cancer. J Exp Clin Cancer Res 34: 60, 2015.

7. Tung PY, Varlakhanova NV and Knoepfler PS: Identification of DPPA4 and DPPA2 as a novel family of pluripotency-related oncogenes. Stem Cells 31: 2330-2342, 2013.

8. Tang Y, Xiao J, Zhao S, Feng Y and Zhang H: A study on construction of plasmid pcDNA3.1 (-) CMV.CD and transfection into laryngeal cancer cell Hep-2. Lin Chuang Er Bi Yan Hou Ke Za Zhi 19: 988-991, 2005 (In Chinese).

9. Peirson SN, Butler JN and Foster RG: Experimental validation of novel and conventional approaches to quantitative real-time PCR data analysis. Nucleic Acids Res 31: e73, 2003.

10. Livak KJ and Schmittgen TD: Analysis of relative gene expression data using real-time quantitative PCR and the 2(-Delta Delta C(T)) method. Methods 25: 402-408, 2001.

11. Chakravarthy H, Boer B, Desler M, Mallanna SK, McKeithan TW and Rizzino A: Identification of DPPA4 and other genes as putative Sox 2:Oct-3/4 target genes using a combination of in silico analysis and transcription-based assays. J Cell Physiol 216: 651-662, 2008

12. Abdul Khalek FJ, Gallicano GI and Mishra L: Colon cancer stem cells. Gastrointest Cancer Res (Suppl 1): S16-S23, 2010.

13. Eramo A, Lotti F, Sette G, Pilozzi E, Biffoni M, Di Virgilio A, Conticello C, Ruco L, Peschle C and De Maria R: Identification and expansion of the tumorigenic lung cancer stem cell population. Cell Death Differ 15: 504, 2008.

14. Jones CL, Stevens BM, D'Alessandro A, Reisz JA, Culp-Hill R, Nemkov T, Pei S, Khan N, Adane B, Ye H, et al: Inhibition of amino acid metabolism selectively targets human leukemia stem cells. Cancer Cell 34: 724-740.e4, 2018.

15. Hanahan D and Weinberg RA: Hallmarks of cancer: The next generation. Cell 144: 646-674, 2011.

16. Lu J, Tan M and Cai Q: The Warburg effect in tumor progression: Mitochondrial oxidative metabolism as an anti-metastasis mechanism. Cancer Lett 356: 156-164, 2015.

17. Lin L, Huang H, Liao W, Ma H, Liu J, Wang L, Huang $\mathrm{N}$ and Liao Y: MACC1 supports human gastric cancer growth under metabolic stress by enhancing the Warburg effect. Oncogene 34 2700-2710, 2015.
18. Christofk HR, Vander Heiden MG, Wu N, Asara JM and Cantley LC: Pyruvate kinase M2 is a phosphotyrosine-binding protein. Nature 452: 181-186, 2008.

19. Mathupala SP, Heese C and Pedersen PL: Glucose catabolism in cancer cells. The type II hexokinase promoter contains functionally active response elements for the tumor suppressor p53. J Biol Chem 272: 22776-22780, 1997.

20. Yoshikawa T, Noguchi Y and Satoh S: Inhibition of IRS-1 phosphorylation and the alterations of GLUT4 in isolated adipocytes from cachectic tumor-bearing rats. Biochem Biophys Res Commun 256: 676-681, 1999.

21. Leiblich A, Cross SS, Catto JW, Phillips JT, Leung HY, Hamdy FC and Rehman I: Lactate dehydrogenase-B is silenced by promoter hypermethylation in human prostate cancer. Oncogene 25: 2953-2960, 2006.

22. Song T, Gan W, Chen J, Huang L, Yin H, He T, Huang H and $\mathrm{Hu}$ X: Antibodies against Clonorchis sinensis LDH could cross-react with LDHB localizing on the plasma membrane of human hepatocarcinoma cell SMMC-7721 and induce apoptosis. Parasitol Res 115: 1595-1603, 2016.

23. McCleland ML, Adler AS, Deming L, Cosino E, Lee L, Blackwood EM, Solon M, Tao J, Li L, Shames D, et al: Lactate dehydrogenase B is required for the growth of KRAS-dependent lung adenocarcinomas. Clin Cancer Res 19: 773-784, 2013.

24. McCleland ML, Adler AS, Shang Y, Hunsaker T, Truong T, Peterson D, Torres E, Li L, Haley B, Stephan JP, et al: An integrated genomic screen identifies LDHB as an essential gene for triple-negative breast cancer. Cancer Res 72: 5812-5823, 2012.

25. Dennison JB, Molina JR, Mitra S, González-Angulo AM, Balko JM, Kuba MG, Sanders ME, Pinto JA, Gómez HL, Arteaga CL, et al: Lactate dehydrogenase B: A metabolic marker of response to neoadjuvant chemotherapy in breast cancer. Clin Cancer Res 19: 3703-3713, 2013.

26. Chen R, Zhou X, Yu Z, Liu J and Huang G: Low expression of LDHB correlates with unfavorable survival in hepatocellular carcinoma: Strobe-compliant article. Medicine (Baltimore) 94: e1583, 2015.

27. Cui J, Quan M, Jiang W, Hu H, Jiao F, Li N, Jin Z, Wang L and Wang Y: Suppressed expression of LDHB promotes pancreatic cancer progression via inducing glycolytic phenotype. Med Oncol 32: 143, 2015.

28. Maekawa M, Taniguchi T, Ishikawa J, Sugimura H, Sugano K and Kanno T: Promoter hypermethylation in cancer silences LDHB, eliminating lactate dehydrogenase isoenzymes 1-4. Clin Chem 49: 1518-1520, 2003

29. Revill K, Wang T, Lachenmayer A, Kojima K, Harrington A, Li J, Hoshida Y, Llovet JM and Powers S: Genome-wide methylation analysis and epigenetic unmasking identify tumor suppressor genes in hepatocellular carcinoma. Gastroenterology 145: 1424-135. e1-e25, 2013.

This work is licensed under a Creative Commons Attribution-NonCommercial-NoDerivatives 4.0 International (CC BY-NC-ND 4.0) License. 